\title{
LA INDUSTRIA DE DEFENSA EN EL MARCO EUROPEO
}

\author{
María Vílchez Vivanco \\ Doctoranda de Universidad de Granada \\ Email: $\underline{\text { mvilchezv@ugr.es }}$
}

\begin{abstract}
RESUMEN: En este artículo se expone cual es la regulación jurídica de la industria de defensa en el marco de la Unión Europea. Esta es una industria que no goza de una buena prensa pero por otro lado tiene un peso específico importante debido a la producción y a la función que ejerce en seguridad.

No hay una legislación específica para este ámbito. La industria de defensa se encuentre enmarcada por la legislación orientada a la industria en general y la que se dirige a regular la seguridad y defensa de la Unión. Su marco depende de los acuerdos de los países miembros.
\end{abstract}

PALABRAS CLAVE: Industria de defensa, Unión Europea, Seguridad, Defensa, Fondo Europeo de la Defensa.

ABSTRACT: This article describes the legal regulation of the defense industry in the framework of the European Union. This industry does not enjoy good press but however it has an important specific weight due to production and the role it plays in security.

There is no specific legislation for this area. The defense industry is framed by the legislation oriented to the industry in general and the one aimed at regulating the security and defense of the Union. Its framework depends on the agreements of the member countries.

KEYWORDS: Defense industry, European Union, Security, Defense, European Defence Fund. 
SUMARIO: I.- Introducción. II.- Regulación de la industria de defensa en el Derecho Originario de la Unión Europea. III.- Regulación de la Industria Europea. IV.- Bases de la industria de defensa europea. 1. Hacia un mercado de la defensa europea. 2. La Agencia Europea de la Defensa. V.- La colaboración en la Unión Europea para una industria de defensa. VI.- Conclusiones.

\section{I.- INTRODUCCIÓN}

La industria de defensa a pesar de ser difícil de definir presenta una serie de características que la diferencian del resto de sectores industriales y que determinan su peculiar naturaleza. De una manera sintética pueden señalarse dos elementos que describen dicha peculiaridad; por un lado los productos, y por otro los clientes que intervienen en este sector. Productores público-privados y compradores eminentemente públicos.

Resulta una tarea compleja y difícil de determinar la coceptualización de los que es una industria de defensa. Como señala PÉREZ FORNIES ${ }^{1}$ son las definiciones que la literatura ofrece acerca de lo que se entiende por esta industria lo que nos sirve de guía. De entre todas ellas son de destacar las de SANDLER y HARTLEY ${ }^{2}$ o la de DUNNE ${ }^{3}$, en la cual se inspiraron los dos anteriores autores.

Siguiendo las recomendaciones de PÉREZ FORNIES, en busca de la definición más adecuada a las necesidades y los propios usos que de ese tipo de industria hacemos, se ha seguido una sencilla y adecuada definición, esta es la que proporciona GARCÍA $\mathrm{ALONSO}^{4}$. Así, en esta, entendemos la industria de defensa como la base industrial y tecnológica de la seguridad y la defensa, incluyendo las plantas de fabricación y la mano de obra experta que se requiere, los centros de I+D, la capacidad exportadora, los conocimientos tecnológicos y el soporte financiero.

Digamos que esta es una definición amplia del concepto, con mayor alcance de entre las que se pueden encontrar en la literatura. En el caso de la investigación es de las

\footnotetext{
1 PEREZ FORNIES, C. en JORDÁN ENAMORADO, J.J., POZO SERRANO, M.P. y BAQUÉS QUESADA, J., La seguridad más allá del Estado: actores no estatales y seguridad internacional. Madrid: Plaza y Valdés, 2011. Pág. 160.

2 SANDLER, T. ; HARTLEY, K. (ed.). Handbook of Defense Economics: Defense in a globalized world. Elsevier, 2007. Págs,. 1141-1142.

${ }^{3}$ DUNNE, J. P. The defense industrial base. Handbook of defense economics, 1995, vol. 1, págs. 399430.

${ }^{4}$ GARCÍA AlONSO, J. M. La base industrial de la defensa en España. Ministerio de Defensa, Secretaría General Técnica, 2010. Págs. 15-16.
} 
más adecuadas ya que un concepto restrictivo no tendría en cuenta la verdadera potencialidad de la industria de defensa europea y de su diversidad.

La definición con la que se va a jugar es esta, la más amplia. Es la más cercana para conocer la realidad europea de este sector. Cómo señala CARMEN GARCÍAVALDÉS DE YRIZAR ${ }^{5}$ desde una perspectiva amplia puede definirse la industria de defensa de manera más acertada. Es clave entender qué es realmente esta industria y no caer en la producción militar estrictamente. La industria de defensa es un sector que va más allá de lo militar. Y no solo esto, cada vez más, la tendencia por la que se trabaja busca un sector más común entre los países y menos militarizado.

La industria de defensa se erige así, como pilar fundamental de la política de defensa de un país, garantía de seguridad de sus ciudadanos al proporcionar los medios necesarios para desarrollar las capacidades precisas que permitan mantener o recuperar la paz, como explica GARCÍA ALONSO. Es en este caso industria de defensa, desde la producción de armamento pesado, hasta la fabricación de textil para el combate, o las tecnologías de vuelo indetectable.

Si nos referimos al espacio de los Estados, la cuestión queda más clara y determinada, el problema es cuando elevamos esta idea a entes supranacionales como la Unión Europea ¿se puede hablar de una industria de defensa a este nivel? ¿en qué marco jurídico se mueve este sector?. Si se puede decir que hay una industria de defensa europea y un marco jurídico que la enmarca, pero ambos se encuentran en proceso, y es en tiempos más recientes cuando se puede decir que tenemos algo más tangible, con mayor sentido de unidad y no como la suma de lo que pudiéramos encontrar a nivel nacional.

Por eso, el tipo de definición tomada engloba bien el concepto con el que se juega a nivel europeo. Nos encontramos con una industria con unas características diferenciadas por sectores, ya que se dedica a los temas de seguridad y defensa en la perspectiva más amplia. La industria, en general, tiene una mayor regulación a nivel europeo, en cambio, en temas de seguridad y defensa cada Estado es el responsable de

\footnotetext{
${ }^{5}$ DE YRIZAR GARCÍA-VALDÉS, C. "La importancia de la industria de defensa en España. En La industria de defensa: el desfase tecnológico entre la Unión Europea y Estados Unidos de América" Instituto Español de Estudios Estratégicos, 2003. p. 159-198. Pág. 161.
} 
sus decisiones, no es una materia común. No se puede hablar de una legislación como tal en temas de industria de defensa.

A esto hay que sumar el carácter del perfil que tiene la oferta y la demanda en este sector ${ }^{6}$. Es de destacar como la demanda viene dada en su inmensa mayoría por los propios gobiernos, mientras que la oferta es del sector público, hay empresas públicas en la producción, pero tiene un carácter mixto, ya que son muchas las empresas privadas que también tienen una iniciativa importante en la producción en el mercado de la defensa.

La industria de defensa es uno de esos escenarios donde es difícil de catalogar y de limitar el área que implica. Tiene una parte muy importante que es similar a cualquier otra industria, pero en cambio tiene la parte referida a seguridad. El enfoque de la política de seguridad y defensa en la Unión Europea es una cuestión delicada, en la que están en juego factores muy sensibles como son la soberanía y la seguridad de los propios países integrantes de la Unión.

La política de seguridad y defensa no se encuentra dentro las materias que son de gestión de la Unión, las iniciativas llevadas a cabo, se encuentran dentro de un espacio de colaboración entre los diferentes Estados miembros. El anclaje dentro del Derecho Originario de la Unión, es clave para conocer la base jurídica de la que se parte en materia de industria de defensa en el espacio europeo.

Con un volumen de negocio de 97.300 millones de euros en 2014, 500.0007 personas directamente empleadas y 1,2 millones de puestos de trabajo indirectos, la industria europea de defensa es un sector industrial importante y destacado. Participa en el 35\% de la producción mundial con 23 millones de euros en exportaciones en 2011. La principal característica de la industria de defensa es que sea reconocida por sus capacidades económicas y el desarrollo de los componentes tecnológicos. Es la gran apuesta en el contexto europeo. Estos son aspectos decisivos para la competitividad industrial de Europa a nivel interno y a nivel externo.

\footnotetext{
${ }^{6}$ MARTÍ SEMPERE, C.; FONFRIA MESA, A. “An Analysis of the Defence Industrial Market Based on Agents". Defence and Peace Economics, 2018. Págs. 1-25.

${ }^{7}$ Datos estimados por el Parlamento Europeo según los cuales de 2011 a 2014 se pasó de 400.000 puesto de trabajo a los 500.000 y el aumento de facturación aumentó en unos 1.300 millones de euros.
} 
Como señala BLANCO en sus análisis, el gasto militar total de la Unión Europea en 2010 se elevó a los 197.000 millones de euros, con un descenso del 3,3\% sobre el año anterior. Se estaba aún al comienzo de la época de descenso de los presupuestos a causa de la crisis económica que se afianzaba. Cuatro Estados miembros siguen estando entre los diez países del mundo que más gasto militar realizan: Reino Unido, Francia, Alemania e Italia. Francia y Reino Unido producen casi la mitad de la demanda del mercado (marcadas por el desarrollo de la industria aeroespacial y naval, respectivamente), seguidas de Alemania (con una fuerte industria de vehículos militares), Italia, España y Suecia (90\% de la producción europea entre esos seis países, que están agrupados en $1 \mathrm{a} \mathrm{LoI}^{8}$ ).

La colaboración se hace necesaria. Los procesos de pooling \& sharing en la industria de defensa europea ya son un imperativo debido a tres factores: el impacto de la crisis económica en los presupuestos de defensa; las lecciones militares de la campaña en Libia y la demanda de la OTAN de una cooperación multilateral en defensa; y la necesaria transformación de la industria de defensa para sobrevivir en un mundo multipolar'.

Todas estas características hacen que sea difícil el determinar un marco estricto jurídico en el que se mueva esta industria en la UE. Diferentes ámbitos la determinan como se va a exponer a continuación.

\section{II.- REGULACIÓN DE LA INDUSTRIA DE DEFENSA EN EL DERECHO ORIGINARIO DE LA UNIÓN EUROPEA}

La capacidad de acción de la Unión Europea en este campo para la adopción de directivas en el tema, se basa en el artículo $352^{10}$ del Tratado de Funcionamiento de la Unión Europea (TFUE).

\footnotetext{
${ }^{8}$ Carta de Intenciones sobre la Industria de Defensa Europea https://www.gov.uk/guidance/letter-ofintent-restructuring-the-european-defence-industry

${ }^{9}$ PINTADO RODRÍGUEZ, C. "Pooling and Sharing y la industria europea de defensa. Viejas ideas para nuevas soluciones". Revista del Instituto Español de Estudios Estratégicos, 104/2013, 29 de octubre de 2013.

${ }^{10} \mathrm{El}$ art. 352 (antiguo artículo 308 TCE) dice así:

1. Cuando se considere necesaria una acción de la Unión en el ámbito de las políticas definidas en los Tratados para alcanzar uno de los objetivos fijados por éstos, sin que se hayan previsto en ellos los
} 
Como se puede apreciar, este es un art. de carácter general. Se prevé para los casos en los que el Tratado de la Unión Europea no contiene explícitamente la acción necesaria para lograr uno de los objetivos de la Unión. Es en sí un art. de regulación general. Se entiende así que no hay una determinación específica, en los Tratados de la Unión, para regular el ámbito de la industria de la defensa. El profesor DIEGO LIÑÁN, se refiere a este artículo como el creado para cubrir la "imprevisión competencial"11 por parte del legislador europeo.

El artículo 173 del Tratado de Funcionamiento de la Unión Europea (TFUE) establece una base jurídica para la política industrial de la Unión Europea. El art. $173^{12}$

poderes de actuación necesarios a tal efecto, el Consejo adoptará las disposiciones adecuadas por unanimidad, a propuesta de la Comisión y previa aprobación del Parlamento Europeo. Cuando el Consejo adopte dichas disposiciones con arreglo a un procedimiento legislativo especial, se pronunciará también por unanimidad, a propuesta de la Comisión y previa aprobación del Parlamento Europeo.

2. La Comisión, en el marco del procedimiento de control del principio de subsidiariedad mencionado en el apartado 3 del artículo 5 del Tratado de la Unión Europea, indicará a los Parlamentos nacionales las propuestas que se basen en el presente artículo.

3. Las medidas basadas en el presente artículo no podrán conllevar armonización alguna de las disposiciones legales y reglamentarias de los Estados miembros cuando los Tratados excluyan dicha armonización.

4.El presente artículo no podrá servir de base para alcanzar objetivos del ámbito de la política exterior y de seguridad común y todo acto adoptado de conformidad con el presente artículo respetará los límites fijados en el párrafo segundo del artículo 40 del Tratado de la Unión Europea.

${ }^{11}$ LIÑÁN NOGUERAS, D.J., SEGURA SERRANO, A. y GARCÍA SEGURA, C., Las crisis politicas y económicas: nuevos escenarios internacionales. Madrid: Tecnos, 2014. Pág. 86.

12173 (antiguo artículo 157 TCE) del Título XVII, dice así:

1. La Unión y los Estados miembros asegurarán la existencia de las condiciones necesarias para la competitividad de la industria de la Unión.

A tal fin, dentro de un sistema de mercados abiertos y competitivos, su acción estará encaminada a:

- acelerar la adaptación de la industria a los cambios estructurales,

- fomentar un entorno favorable a la iniciativa y al desarrollo de las empresas en el conjunto de la Unión, $\mathrm{y}$, en particular, de las pequeñas y medianas empresas,

- fomentar un entorno favorable a la cooperación entre empresas,

- favorecer un mejor aprovechamiento del potencial industrial de las políticas de innovación, de investigación y de desarrollo tecnológico.

2. Los Estados miembros se consultarán mutuamente en colaboración con la Comisión y, siempre que sea necesario, coordinarán sus acciones. La Comisión podrá adoptar cualquier iniciativa adecuada para fomentar dicha coordinación, en particular iniciativas tendentes a establecer orientaciones e indicadores, organizar el intercambio de mejores prácticas y preparar los elementos necesarios para el control y la evaluación periódicos. Se informará cumplidamente al Parlamento Europeo.

3. La Unión contribuirá a alcanzar los objetivos estipulados en el apartado 1 mediante las políticas y actividades que lleva a cabo en virtud de otras disposiciones del presente Tratado. El Parlamento Europeo y el Consejo, con arreglo al procedimiento legislativo ordinario y previa consulta al Comité Económico y Social, podrán tomar medidas específicas destinadas a apoyar las acciones que se lleven a cabo en los Estados miembros a fin de realizar los objetivos contemplados en el apartado 1, con exclusión de toda armonización de las disposiciones legales y reglamentarias de los Estados miembros.

Este título no constituirá una base para el establecimiento por parte de la Unión de medidas que puedan falsear la competencia o incluyan disposiciones fiscales o relativas a los derechos e intereses de los trabajadores asalariados. 
(antiguo artículo 157 TCE) del Título XVII, que trata de la INDUSTRIA, expone la regulación que de la industria en general se debe de desarrollar.

Es de destacar como en este art. se pone de relieve la adaptación, la cooperación, el desarrollo tecnológico y el fomento de la pequeña y mediana empresa, como las bases para generar una industria europea competitiva. Desde el derecho originario se planteaban cuestiones que hasta nuestros días no habían tenido un compromiso más firme de desarrollo.

Estos son factores claves a tener en cuenta en el desarrollo de una industria de defensa europea competitiva. El desarrollo tecnológico es un punto esencial y la cooperación entre los diferentes agentes, el mecanismo que posibilita este modelo de industria que se pretende. Cooperación y tecnología son la base para que se pueda hablar de una industria de defensa europea y competitiva.

En la referencia al propio título del artículo, se expresa, como las medidas que se lleven a cabo en lo industrial, no deben tender a falsear la competencia o actuar de manera que recaiga perjuicio sobre los derechos de los trabajadores. Se persigue un desarrollo industrial europeo que sea competitivo a partir de medios como los que se hablaban al principio del artículo, de la cooperación y desarrollo tecnológico, principalmente.

Sin embargo, el progreso hacia la aplicación de normas para el mercado interior en el tema de equipos de defensa ha sido restringido por el artículo $346(1)^{13}$ del TFUE (antiguo artículo 296 TCE). Una contraposición de intereses.

Es una limitación específica a la producción industrial en temas de seguridad y defensa que establece que cualquier Estado miembro podrá adoptar las medidas que estime necesarias para la protección de los intereses esenciales de su seguridad y que

\footnotetext{
${ }^{13}$ artículo 346 (1) del TFUE (antiguo artículo 296 TCE) dice así:

1. Las disposiciones de los Tratados no obstarán a las normas siguientes:

a) ningún Estado miembro estará obligado a facilitar información cuya divulgación considere contraria a los intereses esenciales de su seguridad;

b) todo Estado miembro podrá adoptar las medidas que estime necesarias para la protección de los intereses esenciales de su seguridad y que se refieran a la producción o al comercio de armas, municiones y material de guerra; estas medidas no deberán alterar las condiciones de competencia en el mercado interior respecto de los productos que no estén destinados a fines específicamente militares.
} 
están conectadas con la producción o al comercio de armas, municiones y material de guerra. Esta es una de las cuestiones más importantes.

Tenemos que tener presente que los temas de seguridad y defensa son el núcleo duro de la soberanía de los Estados, por lo que es difícil el ceder esa competencia a la Unión Europea. Se puede decir que en temas industriales, la seguridad es la excepción. A la vez que es una protección el amparo que da el art. 352 a los Estados sobre la reserva en seguridad, no ayuda a que se genere un mercado interno de la defensa. Son distintas caras de una misma moneda.

Con respecto a la industria europea en general, hay una regulación que busca la cooperación entre los Estados miembros, el desarrollo tecnológico y la competitividad de los productos en el mercado. Digamos pues, que la regulación de la industria europea, se basa en principios que dan pie a una industria de defensa competitiva en lo internacional.

\section{III.- REGULACIÓN DE LA INDUSTRIA EUROPEA}

La política industrial de la UE tiene como objetivo hacer más competitiva la industria europea para que pueda mantener su papel como motor de crecimiento sostenible y el desarrollo del empleo en Europa. Varias estrategias han sido llevadas a cabo con el fin de garantizar mejores condiciones marco para la industria de la Unión Europea, como la comunicación "Para un renacimiento industrial europeo", de enero de $2014^{14}$. Supuso un hito que se ha venido completando con diferentes acciones en la línea de la tecnología, medio ambiente y desarrollo cultural.

Una de las características que definen la política industrial es su horizontalidad. Pretende garantizar unas condiciones marco favorables a la competitividad industrial. También busca integrar un número de políticas comunitarias de otro carácter, como las relativas a comercio, sobre la gestión del mercado interior, el impulso de la investigación y la innovación, el empleo y la calidad de este, la protección del medio

\footnotetext{
${ }^{14}$ Comunicación de la Comisión al Parlamento Europeo, al Consejo, al Comité Económico y Social Europeo y al Comité de las Regiones. Por un renacimiento industrial europeo. Bruselas, 22 de enero de 2014.COM(2014)14 final.

https://eur-lex.europa.eu/legalcontent/ES/TXT/PDF/?uri=CELEX:52014DC0014\&from=EN
} 
ambiente y la salud pública. La política industrial de la Unión está dirigida específicamente a acelerar la adaptación de la industria a los cambios estructurales; fomentar un entorno favorable a la iniciativa y al desarrollo de las empresas de toda la Unión, en particular las pequeñas y medianas empresas; fomentar un entorno favorable a la cooperación entre empresas y favorecer un mejor aprovechamiento del potencial industrial de las políticas de innovación, investigación y desarrollo tecnológico, como se fundamenta en el artículo 173 del TFUE.

Los instrumentos de la política industrial de la UE, que son también los de la política de la empresa, tienen como objetivo fomentar unas condiciones generales en las que los empresarios y las empresas pueden tomar iniciativas y explotar sus ideas y oportunidades como destaca FONFRÍA ${ }^{15}$. No obstante, la política industrial debe tener en cuenta las necesidades y características específicas de cada sector. Los informes anuales sobre la competitividad europea analizan las fortalezas y debilidades de la economía de la Unión en general y su industria en particular, y pueden desencadenar iniciativas políticas intersectoriales o sectoriales.

En julio de 2005, por primera vez, una comunicación de la Comisión, la llamada "Implementación del programa comunitario de Lisboa: Un marco político para fortalecer la industria manufacturera de la UE - hacia un enfoque más integrado de política industrial" ${ }^{\prime 6}$, propuso un nuevo enfoque integrado de política industrial, basada en un programa de trabajo centrado en las iniciativas intersectoriales y sectoriales.

La "Revisión de la política industrial a medio plazo"17 llegó a la conclusión de que las acciones descritas en la Comunicación de 2005 habían beneficiado a industrias de Europa. En consecuencia, se argumentó que el marco debía mantenerse en su lugar, ya que permitiría a la industria responder mejor a los desafíos de la globalización y el cambio climático.

\footnotetext{
${ }^{15}$ ÁLVAREZ, I. ; FONFRÍA; A. ; MARÍN, R.. "El papel de la cooperación en el perfil competitivo de las empresas españolas". Revista Madrid, 2006, no 37. Pág. 4.

${ }^{16}$ Communication from the Commission Implementing the Community Lisbon Programme: A policy framework to strengthen EU manufacturing - towards amore integrated approach for industrial policy. COM (2005) 0474 Bruselas, 5 de octubre de 2005. http://eur-lex.europa.eu/legalcontent/EN/TXT/?uri=CELEX\%3A52005DC0474

${ }^{17}$ Communication from the Commission to the European Parliament, the Council, the european economic and Social Committee and the Committee of the Regions. Mid-term review of industrial policy A contribution to the EU?s Growth and Jobs Strategy. COM (2007) 0374 Bruselas, 4 de julio 2007. http://eur-lex.europa.eu/legal-content/EN/TXT/?uri=CELEX\%3A52007DC0374
} 
La comunicación de la Comisión de 2008 titulada "Producción y Consumo Sostenible y el Plan de Acción de Política Industrial Sostenible" (COM (2008) 0397) ${ }^{18}$ estaba dirigida a la entrega de un paquete integrado de medidas para fomentar el consumo y producción más sostenibles, buscando al tiempo, que la economía europea fuera más competitiva. Equilibrio entre sostenibilidad y competitividad.

En respuesta a los desafíos implicados en asegurar un suministro sostenible de materias primas no energéticas para la economía de la Unión Europea, la Comisión puso en marcha la iniciativa «Materias primas» (COM (2008) 0699) ${ }^{19}$, que trataba de garantizar la igualdad de condiciones en el acceso a recursos en terceros países, mejores condiciones marco para la extracción de materias primas dentro de la Unión, y la reducción del consumo de materias primas de carácter primario, mediante una mayor eficiencia de los recursos y dando promoción el reciclaje. Una posterior comunicación de la Comisión (COM (2011) 0021) ${ }^{20}$ propone reforzar la aplicación de esta iniciativa.

En su comunicación "Preparar nuestro futuro: desarrollo de una estrategia común para las tecnologías clave en la UE” (COM (2009) 0512) ${ }^{21}$, la Comisión declaró que la Unión, fomentaría el despliegue de las tecnologías facilitadoras esenciales (TFE) dentro de su política actual marco, y también sugirió la creación de un grupo de alto nivel de expertos (GAN), que sería responsable del desarrollo de una estrategia común a largo plazo. En su informe final, el GAN propuso once recomendaciones de políticas para el desarrollo y despliegue de las TFE en Europa.

\footnotetext{
${ }^{18}$ Communication from the Commission to the European Parliament, the Council, the european economic and Social Committee and the Committee of the Regions on the Sustainable Consumption and Production and Sustainable Industrial Policy Action Plan. COM (2008) 0397 Bruselas, 16 de julio de 2008. http://eur-lex.europa.eu/legal-content/en/TXT/?uri=celex\%3A52008DC0397

${ }^{19}$ Communication from the Commission to the European Parliament and the Council, The raw materials initiative - meeting our critical needs for growth and jobs in Europe. COM (2008) 0699 Bruselas, 4 de noviembre de 2008. http://eur-lex.europa.eu/legal-content/EN/TXT/?uri=CELEX\%3A52008DC0699

${ }^{20}$ Communication from the Commission to the European Parliament, the Council, the european economic and Social Committee and the Committee of the Regions. A resource-efficient Europe - Flagship initiative under the Europe 2020 Strategy. COM (2011) 0021 Bruselas, 26 de enero de 2011. http://ec.europa.eu/resource-efficient-europe/pdf/resource_efficient_europe_en.pdf

${ }^{21}$ Comunicación de la Comisión al Parlamento Europeo, al Consejo, al Comité Económico y Social Europeo y al Comité de las Regiones. Preparar nuestro futuro: desarrollo de una estrategia común en la UE para las tecnologías facilitadoras esenciales. COM (2009) 0512 Bruselas, 30 de septiembre de 2009. http://eur-lex.europa.eu/legal-content/ES/TXT/?uri=URISERV\%3Aet0002
} 


\section{IV.- BASES DE LA INDUSTRIA DE DEFENSA EUROPEA}

Uno de los objetivos principales como indicaba anteriormente, de la política de la industria de defensa de la UE es el desarrollo de una base de Tecnología Europea de Defensa y Base Industrial ${ }^{22}$ (TEDBI). Esta base se vería reforzada si se estableciese un mercado de equipos de defensa europea integrada.

En julio de 2006 se lanza el Régimen Intergubernamental ${ }^{23}$ para fomentar la competencia en el mercado europeo de equipos de defensa. Este régimen intergubernamental es de carácter voluntario y se opera sobre la base de un código de conducta sobre cómo realizar contratos públicos de defensa, el cual se apoya en un sistema de información y seguimiento para ayudar así a asegurar la transparencia y rendición de cuentas mutua entre los Estados miembros. Este es un hito importante y destacado.

Otro elemento importante es el "Código de Buenas Prácticas en la Cadena de Suministro" (de mayo de 2005) ${ }^{24}$. La estandarización de los equipos de defensa es importante para la integración de los mercados nacionales, permitiendo la colaboración y cooperación en la producción. Se han tomado medidas en este sentido, como la creación por la Comisión de un manual europeo de los contratos de defensa ${ }^{25}$, que presenta los estándares y referencias a los compradores públicos, la mejor manera de especificar en los contratos de defensa.

El manual fue pensado para ser manejado por la Agencia Europea de Defensa (AED). La AED ha desarrollado un sistema de información propio, dentro de su portal, para una normalización de los materiales de defensa europea a mayor alcance. El

\footnotetext{
22 Propuesta de Resolución del Parlamento Europeo sobre la base tecnológica e industrial de la defensa europea. (2013/2125(INI)) 30 de octubre de 2013.

http://www.europarl.europa.eu/sides/getDoc.do?pubRef=-//EP//TEXT+REPORT+A7-2013 $0358+0+\mathrm{DOC}+\mathrm{XML}+\mathrm{V} 0 / / \mathrm{ES}$

23 Informe de la Comisión. La Unión Europea y los Estados Unidos Socios mundiales con responsabilidades mundiales http://eeas.europa.eu/us/docs/infopack_06_es.pdf

${ }^{24}$ Comunicación de la Comisión al Parlamento Europeo, al Consejo, al Comité Económico y Social Europeo y al Comité de las Regiones Hacer frente a las prácticas comerciales desleales en la cadena de suministro alimentario entre empresas, $\operatorname{COM}(2014) 472$, Estrasburgo, 15 de julio de 2014. http://www.magrama.gob.es/es/alimentacion/temas/ley-de-medidas-para-mejorar-el-funcionamiento-dela-cadena-alimentaria/com2014472hacerfrentealaspracticascomercialesdesleales_tcm7-405101.pdf ${ }^{25} \mathrm{http}$ ://simap.ted.europa.eu/documents/10184/36234/cpv_2008_guide_es.pdf. Este es producto de una normalización del modelo de acuerdo más habitual que se realizaba en las transacciones.
} 
objetivo es proporcionar estándares para publicidad de materiales, que se van a desarrollar o que vayan a sufrir una modificación sustantiva.

Se pretende crear una hoja de ruta por la Comisión y la AED para el desarrollo de estándares de la industria de defensa, así como de las opciones para bajar los costos de la certificación militar, incluyendo, claro está en todo esto, el reconocimiento mutuo entre los Estados miembros.

La Comisión adoptó, el 24 de julio de 2013, una comunicación ${ }^{26}$ que contiene un plan de acción para mejorar la eficiencia y la competitividad de la Industria Europea de Defensa. Las iniciativas abarcaban las áreas de: mercado interior, política industrial, investigación e innovación, capacidades, espacio, energía y comercio internacional. Con respecto a los contratos de defensa. El 19 de diciembre de $2013^{27}$, el Consejo Europeo debatió la decisión adoptada y concluyó identificar sobre la Política Común de Seguridad y Defensa (PCSD) una serie de acciones prioritarias.

\section{Hacia un mercado de la defensa europea}

En septiembre de 2004, la Comisión presentó un Libro Verde ${ }^{28}$ sobre los contratos públicos de defensa (COM (2004) 0608) ${ }^{29}$ con el objetivo de contribuir a la creación gradual de un mercado de equipos de defensa europea (MEDE) entre los Estados miembros en busca de una mayor transparencia y accesibilidad.

El Libro Verde fue un paso más en la estrategia de creación de una política de equipos de defensa de la Unión Europea. Tras muchos informes y reuniones de los grupos de trabajo fue aprobado por la Comisión a principios de 2003. El objetivo perseguido era

\footnotetext{
${ }^{26}$ Comunicación de la Comisión al Parlamento Europeo, al Consejo, al Comité Económico y Social Europeo y al Comité de las Regiones. Hacia un sector de seguridad y defensa más competitivo y eficiente. $\operatorname{COM}(2013) \quad 542$ Bruselas, 24 de julio de 2013. http://eur-lex.europa.eu/legalcontent/ES/TXT/?uri=CELEX\%3A52014DC0387

${ }^{27}$ Consejo Europeo 19 y 20 de diciembre de 2013 Parte I, puntos 1-22 de las Conclusiones del Consejo Europeo (ya adoptadas) Bruselas, 19 de diciembre de 2013. http://www.consilium.europa.eu/uedocs/cms_data/docs/pressdata/es/ec/140220.pdf

${ }^{28}$ Para conocer más, ver HALL, D., et al. PPP s: a critique of the Green Paper. 2004, BLAUBERGER, M.; WEISS, M. “'If you can't beat me, join me!'How the Commission pushed and pulled member states into legislating defence procurement". Journal of European Public Policy, 2013, vol. 20, no 8, p. 1120-1138, y "Defence procurement in the European Union: The current debate". European Union Institute for Security Studies, 2005.

${ }_{29}$ Green Paper - Defence procurement COM/2004/0608, septiembre de 2004. http://eurlex.europa.eu/LexUriServ/LexUriServ.do?uri=CELEX:52004DC0608:EN:HTM
} 
lograr un uso más eficiente de los recursos en el ámbito de la defensa y elevar la competitividad de la industria en Europa, así como ayudar a lograr mejoras en equipamiento militar dentro del espacio que supone la política de seguridad y defensa en la Unión.

Los contratos de adquisiciones militares y de seguridad se caracterizan por su complejidad y sensibilidad. Por lo tanto, las normas de contratación pública habituales (Directiva 2004/18 / $\mathrm{CE}^{30}$ ) eran poco adecuadas ya que no atienden a lo delicado de las cuestiones que se tratan en temas de seguridad y defensa. La directiva 2009/81/ CE ${ }^{31}$ introdujo reglas más justas y transparentes para las adquisiciones en defensa, lo que debería hacer más fácil para las empresas de defensa el acceder a los mercados de defensa del resto de los Estados miembros.

La directiva 2009/43 / $\mathrm{CE}^{32}$ relativa a las transferencias dentro de la Unión Europea de productos relacionados con la defensa simplifica y armoniza las condiciones y procedimientos para la transferencia de estos productos en toda la Unión. Se crea un sistema uniforme y transparente de los tres tipos de licencias: generales, globales e individuales dentro de esta directiva. Estas iniciativas van encaminadas al desarrollo de un mercado y un marco para la industria de defensa de carácter europeo.

\section{La Agencia Europea de la Defensa}

En 2004, aparece la Agencia Europea de Defensa (AED), la cual contribuye activamente al desarrollo de esta industria. Este sector se enfrenta actualmente a retos importantes $^{33}$ : la disminución en el gasto de defensa de la Unión afecta a la inversión en

\footnotetext{
${ }^{30}$ Diario Oficial de la Unión Europea. Directiva 2004/18 / CE Parlamento Europeo y del Consejo de 31 de marzo de 2004 sobre coordinación de los procedimientos de adjudicación de los contratos públicos de obras, de suministro y de servicios.

http://eur-lex.europa.eu/legal-content/ES/TXT/?uri=URISERV\%3Al22009

${ }^{31}$ Diario Oficial de la Unión Europea Directiva 2009/81/CE del parlamento Europeo y del Consejo de 13 de julio de 2009 sobre coordinación de los procedimientos de adjudicación de determinados contratos de obras, de suministro y de servicios por las entidades o poderes adjudicadores en los ámbitos de la defensa y la seguridad, y por la que se modifican las Directivas 2004/17/CE y 2004/18/CE. http://eurlex.europa.eu/legal-content/ES/TXT/?uri=CELEX\%3A32009L0081

32 Diario Oficial de la Unión Europea Directiva 2009/43/CE del Parlamento Europeo y del Consejo de 6 de mayo de 2009 sobre la simplificación de los términos y las condiciones de las transferencias de productos relacionados con la defensa dentro de la Comunidad. https://www.boe.es/doue/2009/146/L00001-00036.pdf

${ }^{33}$ PÉREZ-FORNIÉS, C., et al. "Gasto en defensa y renta en los países de la Alianza Atlántica" (1960-
} 
investigación e innovación, la creciente competencia en el mercado mundial, la aplicación del marco regulador del mercado interior para la defensa y el apoyo a las PYMEs relacionadas con la defensa en tiempos de austeridad económica.

La Agencia Europea de Defensa se estableció en virtud de una acción común del Consejo de Ministros el 12 de julio de $2004^{34}$, para apoyar a los Estados miembros y el Consejo en su esfuerzo por mejorar las capacidades de defensa europeas en el campo de la gestión de crisis y respaldar la Seguridad y Defensa europea en su situación actual de desarrollo en el futuro.

El 12 de julio de $2011^{35}$, el Consejo adoptó una decisión que define el estatuto, la sede y la forma de funcionamiento de la Agencia Europea de Defensa. Esta decisión del Consejo sustituyó a la Acción Común previa.

La Agencia Europea de Defensa, dentro de la misión general que esta tiene establecido en la Acción Común, se le atribuyen cuatro funciones:

- El desarrollo de las capacidades de defensa.

- La promoción de Investigación y Tecnología en defensa.

- Promoción de la cooperación de armamento.

- La creación de una defensa europea competitiva del mercado del equipo y el fortalecimiento de la Tecnológica de Defensa y la base industrial.

AED actúa como un catalizador, digámoslo así, que promueve la colaboración, pone en marcha nuevas iniciativas y presenta soluciones para mejorar las capacidades de defensa a nivel europeo. Es el lugar donde los Estados miembros que se encuentran dispuestos a desarrollar capacidades en cooperación en temas de defensa, puedan hacerlo. También es un facilitador clave en el desarrollo de las capacidades necesarias para apoyar la Política Común de Seguridad y Defensa de la Unión.

\footnotetext{
2002). Hacienda Pública Española, 2004, no 3, p. 137-153.

${ }^{34}$ Acción Común 2004/551/PESC del Consejo de 12 de julio de 2004 relativa a la creación de la Agencia Europea de Defensa. http://eur-lex.europa.eu/legal-content/ES/ALL/?uri=CELEX:32004E0551

35 Diario Oficial de la Unión Europea Decisión 2011/411/PESC DEL CONSEJO de 12 de julio de 2011 por la que se determinan el estatuto, la sede y la forma de funcionamiento de la Agencia Europea de Defensa y por la que se deroga la Acción Común 2004/551/PESC. http://eur-lex.europa.eu/legalcontent/ES/TXT/?uri=CELEX\%3A32011D0411
} 
La Agencia Europea de Defensa ${ }^{36}$ es una agencia intergubernamental del Consejo Europeo. Actualmente la componen 27 países, todos los Estados miembros de la Unión Europea, excepto Dinamarca.

La misión de la Agencia, es apoyar a los Estados miembros y al Consejo en su esfuerzo por mejorar las capacidades de defensa europeas. También es muy importante el papel que tiene en la cooperación con los Estados miembros. Esta colaboración es muy estrecha; ya sea en el nivel superior a través de la Junta Directiva que establece las prioridades de la AED o en el nivel de trabajo de los equipos de expertos. De hecho, la AED conecta actualmente alrededor de 4.000 expertos nacionales basadas en proyectos de cooperación en defensa. La Agencia recibe al mes alrededor de 1.000 visitantes en su sede en Bruselas. Todo esto siempre asegurándose de que los proyectos están unidos a las necesidades de los Estados miembros y al desarrollo internacional.

La AED es la gran apuesta europea para la creación de un mercado de base tecnológica altamente desarrollado de carácter europeo. La AED sería el núcleo de encuentro de todas las partes, llevando a la coordinación. Un eje entorno al que desarrollar un mercado de defensa.

En la reunión de la Comisión de diciembre de 2013, el Consejo Europeo animó a los Estados a aumentar las inversiones en investigación, pero de forma cooperativa con otros países. Este tipo de programas serian coordinados por la AED, llevando ésta a cabo iniciativas para estimular el mercado y la investigación de doble uso.

Se intenta reproducir lo que ya sucedió con la Letter of Intent ${ }^{37}$ (LoI). Se pretende un escenario de relación y colaboración entre los estados. Este fue un acuerdo de intenciones de Francia, Alemania, Italia, Suecia, Reino Unido y España, sobre la industria de defensa europea. Su objetivo era crear el marco político y jurídico necesario para facilitar la reestructuración industrial con el fin de promover una defensa europea más competitiva $\mathrm{y}$ fuerte de base tecnológica e industrial (BTID) ${ }^{38}$ en el mercado de defensa global.

\footnotetext{
${ }^{36}$ http://www.eda.europa.eu/ ver también Acción Común 2004/551/PESC del Consejo de 12 de julio de 2004 relativa a la creación de la Agencia Europea de Defensa. http://eur-lex.europa.eu/legalcontent/ES/ALL/?uri=CELEX:32004E0551

${ }^{37}$ Carta de Intenciones sobre la Industria de Defensa Europea se firmó el 27 de julio de 2000 por los Ministros de Defensa de los Estados parte. https://www.gov.uk/guidance/letter-of-intent-restructuringthe-european-defence-industry

${ }^{38}$ Informe sobre la base tecnológica e industrial de la defensa europea (2013/2125(INI)) Comisión de
} 


\section{V.- LA COLABORACIÓN EN LA UNIÓN EUROPEA PARA UNA INDUSTRIA DE DEFENSA}

Las cuestiones que atañen a temas de seguridad y defensa de la Unión son en los últimos tiempos fundamentales. Expone ROLDÁN BARBERO que un nuevo buque insignia, y una nueva batalla política por librar, recorre Europa: el robustecimiento de su política de defensa ${ }^{39}$. En los últimos tres años se ha avanzado más por una seguridad y defensa común que en el resto. El avance en la PESC lleva a que vaya configurando cada vez más de forma más clara una industria de defensa europea.

El área de la política de defensa es principalmente el dominio de los estados nacionales, como se ha expuesto en la investigación. La principal alianza militar en Europa sigue siendo la intergubernamental Organización del Tratado del Atlántico Norte (OTAN), que incluye 22 de los Estados miembros de la UE, junto con cuatro países no comunitarios europeos, Albania, Islandia, Turquía y Noruega, así como los Estados Unidos y Canadá ${ }^{40}$. A pesar de esto, los pasos que se están dando en la línea de una defensa con recursos europeos son grandes.

El artículo 42 del Tratado de la Unión Europea ${ }^{41}$ prevé la integración militar de manera sustancial dentro del marco institucional de la Unión. La integración completa es una opción que requiere la unanimidad en el Consejo Europeo de Jefes de Estado o de Gobierno. Por ahora permanece paralizado políticamente, teniendo en cuenta la postura crítica del Reino Unido en particular.

Asuntos Exteriores de 29 de octubre de 2013. http://www.europarl.europa.eu/sides/getDoc.do?pubRef=//EP//TEXT+REPORT+A7-2013-0358+0+DOC+XML+V0//ES

${ }^{39}$ ROLDÁN BARBERO, J "La Europa de la defensa pasa a la ofensiva", Revista General de Derecho Europeo, núm. 43, 2017.

40 Aquí se pueden consultar los Estados miembros con el año de entrada y el modo de ratificación http://www.nato.int/cps/en/natohq/nato_countries.htm

41 art. 42.2TUE dice:

2. La política común de seguridad y defensa incluirá la definición progresiva de una política común de defensa de la Unión. Ésta conducirá a una defensa común una vez que el Consejo Europeo lo haya decidido por unanimidad. En este caso, el Consejo Europeo recomendará a los Estados miembros que adopten una decisión en este sentido de conformidad con sus respectivas normas constitucionales.

La política de la Unión con arreglo a la presente sección no afectará al carácter específíco de la política de seguridad y de defensa de determinados Estados miembros, respetará las obligaciones derivadas del Tratado del Atlántico Norte para determinados Estados miembros que consideran que su defensa común se realiza dentro de la Organización del Tratado del Atlántico Norte (OTAN) y será compatible con la política común de seguridad y de defensa establecida en dicho marco. 
El Tratado de Lisboa ha añadido la posibilidad de que los miembros cuyas capacidades militares cumplan criterios más elevados y que hayan suscrito compromisos vinculantes, pueden realizar las misiones más exigentes. Estableciendo una cooperación estructurada permanente en el marco de la UE (PSCD).

Una de las primeras acciones fue la propuesta de la Comisión en noviembre de 2006 de un Fondo Europeo de Defensa y otras actuaciones para ayudar a los Estados miembros a hacer más eficiente el gasto en capacidades de defensa conjuntas. Se hacía necesario reforzar la seguridad de los ciudadanos europeos y fomentar un base industrial competitiva e innovadora llegando así al Plan de Acción Europeo de la Defensa $\operatorname{COM}(2016) 0950^{42}$. Para el próximo marco financiero plurianual de la Unión (20212027), la Comisión pretende proponer un programa europeo específico de investigación en materia de defensa, con un presupuesto estimado de 500 millones EUR anuales. ${ }^{43}$

Uno de los principios de este protocolo busca cooperar y armonizar los requisitos y recursos de uso en los campos relacionados con la adquisición de equipos de defensa, la investigación, la financiación y la utilización, en particular los programas e iniciativas de la Agencia Europea de Defensa (por ejemplo, se ha establecido un código de conducta sobre contratos públicos de defensa). Esta cuestión afecta de lleno a la industria de defensa europea.

En dos Resoluciones adoptadas en mayo de 2015 y abril de 2016, el Parlamento pedía una política europea exterior y de seguridad eficaz y ambiciosa basada en una visión compartida de los principales intereses europeos. Instaba a los Estados miembros a definir los objetivos de esta política a partir de intereses comunes compartidos, y defendía la adopción de un Libro Blanco sobre la defensa de la Unión sobre la base de una estrategia global. El Parlamento Europeo abogaba por una estrecha cooperación en materia de PCSD y defensa entre los Estados miembros. En noviembre de 2016, el Parlamento aprobó una resolución sobre la Unión Europea de Defensa en la que se proponía su creación urgente.

42 COMMUNICACIÓN DE LA COMISIÓN AL PARLAMENTO EUROPEO, AL CONSEJO EUROPEO, AL CONSEJO, AL COMITÉ ECONÓMICO Y SOCIAL EUROPEO Y AL COMITE DE LAS REGIONES Plan de Acción Europeo de la Defensa https://eurlex.europa.eu/LexUriServ/LexUriServ.do?uri=COM:2016:0950:FIN:ES:PDF

${ }^{43}$ FED http://europa.eu/rapid/press-release_IP-17-1508_es.htm 
El 11 de diciembre de 2017, el Consejo adoptó la Decisión (PESC) 2017/2315 ${ }^{44}$. En esta Decisión se establecen los modelos para una cooperación estructurada permanente (CEP) en materia de seguridad y defensa. En los modelos de colaboración está el de generar una colaboración en producción de equipos de defensa. Este es el avance en la línea de conseguir un mercado europeo de la defensa.

El 25 de junio de 2018 la Decisión PESC 2018/909 establece un conjunto de reglas de gobernanza comunes para proyectos de CEP. La cooperación funciona entre los Estados llegando a establecerse un protocolo ${ }^{45}$ establecido por el art. 42 del TUE. En dicho protocolo se establece el funcionamiento de la cooperación.

El protocolo sobre la CEP expone que estará abierta a todos los Estados miembros a participar, en caso necesario, en el desarrollo de programas comunes o europeos de equipos de gran envergadura en el marco de la Agencia Europea de Defensa. Con este protocolo la AED comienza a cumplir realmente los fines para los que había nacido. Se le hace además a AED parte en la evaluación periódica de las contribuciones de los Estados miembros, se le da así poder de acción.

Todas estas iniciativas en cooperación necesitaban una financiación económica. Estamos en el camino de un mercado común en defensa y de una industria de defensa en la que la base tecnológica nazca por la colaboración entre los países miembros. Por las necesidades de carácter económico y de gestión aparece el Plan de Acción Europeo de Defensa: hacia un Fondo Europeo del Defensa (FED) a finales de 2016 ${ }^{46}$. Sus fundamentos son la financiación de investigación y el desarrollo de capacidades militares europeas por parte de la UE, con el fin de alcanzar una eficiencia en el gasto en defensa que se aproxime a la de otros grandes actores en la escena internacional ${ }^{47}$.

\footnotetext{
${ }^{44}$ Decisión (PESC) 2018/909 del Consejo de 25 de junio de 2018 por la que se establece un conjunto de reglas de gobernanza comunes para proyectos de la cooperación estructurada permanente (CEP) https://eur-lex.europa.eu/legal-content/ES/TXT/PDF/?uri=CELEX:32018D0909\&from=ES

45 Protocolo ( $\mathrm{N}^{\mathrm{O}}$ 10) sobre la Cooperación Estructurada Permanente establecida por el artículo 42 del Tratado de la Unión Europea

https://eur-lex.europa.eu/legal-content/ES/TXT/?uri=CELEX\%3A12012M\%2FPRO\%2F 10

${ }^{46}$ Plan de Acción Europeo de Defensa: hacia un Fondo Europeo de Defensa http://europa.eu/rapid/pressrelease_IP-16-4088_es.htm

${ }^{47}$ IGLESSIAS, G. y ÁLVAREZ, J. "El Plan de Acción Europeo de Defensa, un impulso a la industria de defensa y un medio de integración de la Unión Europea”. Boletín del Instituto Español de Estudios Estratégicos, 2018, no 9, p. 385-402.
} 
JEAN-CLAUDE JUNCKER en el discurso establecido sobre el estado de la Unión de $2016^{48}$, puso de relieve la importancia de una Europa fuerte capaz de defender $\mathrm{y}$ proteger a sus ciudadanos en el interior y en el exterior. Europa apostaba por seguridad y defensa a nivel interno y eso requiere recursos. Más adelante el Reglamento (UE) 2017/2396 del Parlamento Europeo y del Consejo de 13 de diciembre de $2017^{49}$ vigente hoy, ampliaba el FED. El camino que indicaba JUNCKER en su discurso es el que se está siguiendo.

El FED nace con la intención de reducir las duplicaciones de gastos y gestionar mejor los recursos. Este consta de dos subsecciones: por un lado investigación y desarrollo y por otro adquisición. Es la primera vez que la UE financiará investigación orientada a la defensa. Sin duda este fondo es el gran golpe de efecto para la industria de defensa, a través de él se financian los medios tecnológicos que constituye la base de esta industria. En los comunicados de prensa de la comisión $^{50}$ se habla de una financiación de 5.500 millones euros. No estamos hablando de un fondo vacío, es un fondo con recursos.

El coste económico de un fondo común se podría entender como un aumento del gasto para los Estados miembros, pero la información que la UE ha extraído ${ }^{51}$ concluye que la cooperación disminuiría el gasto por país. Los costes de los equipos de defensa, incluidos los costes de desarrollo, están aumentando más rápido que los presupuestos de defensa. Se calcula que la falta de cooperación entre los Estados miembros en el ámbito de la defensa y la seguridad supone anualmente entre 25.000 millones y 100.000 millones de euros ${ }^{52}$. El $80 \%$ de las adquisiciones y más del $90 \%$ de Investigación y Tecnología se realizan a nivel nacional ${ }^{53}$. Hasta el $30 \%$ de los gastos de defensa anuales podrían salvarse mediante la puesta en común de las adquisiciones ${ }^{54}$.

48 Estado de la Unión https://ec.europa.eu/commission/priorities/state-union-speeches/state-union2016_es

${ }^{49}$ Reglamento (UE) 2015/1017 del Parlamento Europeo y del Consejo de 25 de junio de 2015 relativo al Fondo Europeo para Inversiones Estratégicas, al Centro Europeo de Asesoramiento para la Inversión y al Portal Europeo de Proyectos de Inversión, y por el que se modifican los Reglamentos (UE) no 1291/2013 y (UE) no 1316/2013 - el Fondo Europeo para Inversiones Estratégicas https://eur-lex.europa.eu/legalcontent/ES/ALL/?uri=CELEX:32015R1017

${ }^{50}$ Comisión Europea - Comunicado de prensa. Bruselas, 7 de junio de 2017. http://europa.eu/rapid/pressrelease IP-17-1508 es.htm

${ }^{51}$ Comisión Europea - Hoja informativa http://europa.eu/rapid/press-release_MEMO-17-1476_en.htm

${ }^{52}$ EPRS, Parlamento Europeo, 2013

53 Datos de la Agencia Europea de Defensa en su informe de 2014. https://www.eda.europa.eu/info- 
Todas estas iniciativas han tenido una acogida positiva por parte de los Estados y de las instituciones. Más allá de temas de seguridad y defensa, se trata de reforzar una industria europea de defensa, que interactúe con la política industrial general de la UE, que genere puestos de trabajo y que gane la batalla de la innovación y tecnología al comienzo de una cuarta revolución industrial presidida por la robótica en la que la Unión no puede quedarse rezagada como señala el profesor ROLDÁN ${ }^{55}$.

No podemos olvidar que el programa de orientación para investigación en el ámbito civil de la Unión para la investigación y la innovación, Horizonte 2020 (20142020), se ha ampliado para abarcar los proyectos de «doble uso». Uno de sus retos «Sociedades seguras» es fomentar la investigación para proteger la libertad y la seguridad de Europa y sus ciudadanos. Por medio de una implicación que va más allá de lo estrictamente militar. El presupuesto que se ha asignado a Sociedades seguras para el período 2014-2020 es de 1700 millones EUR, lo que supone el 2,2 \% de los fondos de Horizonte $2020^{56}$. Esto es indicador de la implicación y las necesidades que se van descubriendo. Las cuestiones de seguridad en la Unión no solo se ven relacionadas con la PESC.

Como define el general FRANCISCO GAN PAMPOLS las amenazas ya no proviene exclusivamente de los estados, sino de múltiples actores, y se producen cambios en los elementos que hasta entonces habían definido las guerras y que, en el nuevo contexto, se difuminan: ya no existe una distinción clara entre lo público (relativo al Estado ) y lo privado (las actividades no estatales), entre lo interno y lo externo, entre lo económico y lo político, entre lo civil y lo militar y entre los que emplean la fuerza y los no combatientes. Las amenazas están interconectadas y las fronteras de la seguridad se han desdibujado ${ }^{57}$.

Estos cambios en las amenazas han modificado las nociones sobre seguridad, ampliando sus acepciones e instaurando un nuevo paradigma. Puede que esa sea una de

\footnotetext{
hub/defence-data-portal.

54 Informe de seguridad de Munich 2017. http://www.dsn.gob.es/es/actualidad/sala-prensa/munichsecurity-report-2017

${ }^{55}$ ROLDÁN BARBERO, J "La Europa ...” op cit.

56 “Sociedades Seguras" proyecto Horizonte 2020

http://eshorizonte2020.cdti.es/index.asp?MP=87\&MS=721\&MN=2

${ }^{57}$ GAN PAMPOLS, F. J. "La conciencia intercultural aplicada en un escenario asiático: Afganistán”. En La conciencia intercultural ("Cross-cultural awareness") en la resolución de crisis y conflictos. Universidad de Granada, 2013. p. 173-196. Pág.183.
} 
las razones por la que en los últimos años se ha acelerado un proyecto común de defensa, en el que se está enmarcando una industria de defensa con un verdadero carácter europeo. La seguridad se considera como un bien público que el Estado debe garantizar con todos los instrumentos que tiene a su alcance.

\section{VI.- CONCLUSIONES}

Tras el análisis realizado sobre el marco jurídico en el que se encuentra la Industria Europea de Defensa son muy claras las ideas que se pueden extraer.

La industria de defensa no tiene una legislación específica. Se encuentra ubicada entre la legislación en materia industrial y la que existe en temas de seguridad. Lo relativo a lo industrial queda claro, pero es en el ámbito de la seguridad y defensa dónde esta industria adquiere su carácter propio y dónde surgen las dudas con respecto a su definición y funcionamiento.

Las cuestiones relativas a la defensa se encuentran contempladas en el Derecho originario de la Unión Europea. Están recogidas, pero de manera que se reconoce a los Estados como titulares de esa competencia. Los futuros avances que se produzcan en relación con este tema en la Unión requerirán indudablemente un acuerdo previo de éstos. Los temas de seguridad son el núcleo esencial de la configuración del Estado, no son materia común.

En esta industria la tecnología es una cuestión fundamental. Por parte de la Unión se ha fomentado la creación de un Mercado Común de la Defensa en el que haya una base tecnológica importante. Se considera necesario el desarrollo de este marcado tanto por cuestiones securitarias como por el desarrollo industrial.

Se ha fomentado que ese mercado no tenga un carácter estrictamente militar. El fomento del desarrollo de las tecnologías de doble uso ha llevado a iniciativas comunes civiles-militares en las que se consigue una mayor eficiencia en el uso de los recursos. Esto también ha hecho que cambie el carácter de la industria de la defensa, apartando a esta un poco de lo estrictamente militar y acercándose al sector civil. 
Para el fomento de este mercado se ha implementado la Agencia Europea de la Defensa. Esta Agencia tiene carácter intergubernamental y busca la colaboración entre los diferentes Estados para coordinar y disminuir esfuerzos entre las diferentes iniciativas. Por ahora la AED no ha tenido el éxito que pretendía, tenemos que tener en cuenta que en los últimos años la reducción del gasto tanto en defensa como en investigación en todos los países de la UE no ha ayudado en la situación.

A pesar de ello, desde la UE se insta a que sea la AED la que coordine las diferentes iniciativas. En la gran apuesta civil de la UE como es el Proyecto Horizonte 2020 hay una partida específica para seguridad y es de ahí desde dónde se insta al desarrollo de tecnologías de doble uso, aunque el gran fondo de recursos es el desarrollo del Fondo Europeo para la Defensa. El FED cuenta con recursos que hacen posible el desarrollo de una industria europea de defensa. La sección "Investigación" del Fondo Europeo de Defensa ya dispone de 500 millones de euros.

El marco jurídico europeo para la Industria Europea de Defensa nos da a entender que esta es una industria que cada día tendrá más de industria de base tecnológica y menos de defensa, ya que la defensa se diluirá y se encontrará presente en muchos más sectores. La regulación de todo ello seguirá dependiendo mucho de la postura de los Estados miembros, ya que para bien o para mal la seguridad y defensa escapan de las competencias de la UE.

Seguridad, defensa, colaboración y tecnología serán las claves a tener en cuenta en el futuro de este sector y de su regulación. 